\title{
Effects of sentence ordering on thematic decisions to remember and forget prose
}

\author{
RALPH E. GEISELMAN \\ University of California, Los Angeles, California 90024
}

\begin{abstract}
Sentences from each of two different passages were intermixed and presented to subjects auditorily. During each intersentence interval, the subjects made a to-beremembered (TBR) vs to-be-forgotten (TBF) decision on the basis of theme membership and then selectively rehearsed the TBR sentences for later recall. Presenting either the TBR or TBF sentences in a logical order facilitated sentence recall of both types but had little effect on recognition. The within-subject relationships between decision time and recall were consistent with the between-subject effects of presenting either passage in a logical order on the recall of the remaining passage. Shorter decision times were associated with greater TBR recall but longer decision times were associated with greater TBF recall. It was concluded that processing during the decision phase was different from the maintenance rehearsal found during the TBR-TBF cue-delay interval in directed-forgetting tasks. Ordering of the TBF message did not affect processing if its general theme was not known.
\end{abstract}

Selective forgetting has historically been assigned an integral role in the processing of to-be-remembered (TBR) information. In 1882, Ribot wrote, "Oblivion... is no malady of memory, but a condition of its health and its life" (p. 61). William James (1890) concurred with Ribot in that, "If we remembered everything, we should on most occasions be as ill off as if we remembered nothing" (p. 680). One method of studying the memory mechanisms involved in selective forgetting is to direct subjects to forget intentionally some bits of presented information (to-be-forgotten, or TBF, information) and to remember other bits of information (TBR information). Bjork's $(1970,1972)$ theory of directed forgetting states that the effectiveness of such artificial cues to forget is a direct function of the extent to which the experimental procedure permits the differential grouping of the TBR inputs from the TBF inputs in memory and the selective rehearsal of the TBR inputs.

A more typical instance of selective forgetting occurs when the TBR vs TBF decisions are made on the basis of differential semantic properties of the inputs rather than on the basis of artificial postinput cues. Semantic positive forgetting (Geiselman, 1975; Geiselman \& Riehle, 1975) refers to making TBR vs TBF judgments for sequentially presented sentences on the basis of the semantic content of the sentences. In these two studies, two sets of sentences, each with a distinctly different general theme, were presented to subjects in an intermixed fashion with 7-sec empty intersentence intervals. The subjects were told both themes and also that they would be tested only for their retention of the sentences

This research was conducted while the author was at the Department of Psychology, Ohio University. Requests for reprints should be sent to the author, Department of Psychology, University of California at Los Angeles, Los Angeles, California 90024. about an experimenter-designed theme (the TBR theme). The subject's task was to first decide whether a presented item was TBR or TBF and then use the remaining intersentence time to code the item into longterm memory and/or rehearse the previously presented TBR items. Making the TBR vs TBF decision will be called the decision phase of the semantic positiveforgetting task and the remainder of the intersentence interval will be called the selective-rehearsal phase. These two phases of the task are illustrated in Figure 1.

The semantic positive-forgetting task is strikingly similar to tasks involving goal-directed learning (see Rothkopf, 1976, for a review). Numerous studies have

THE SEMANTIC POSITIVE-FORGETTING TASK

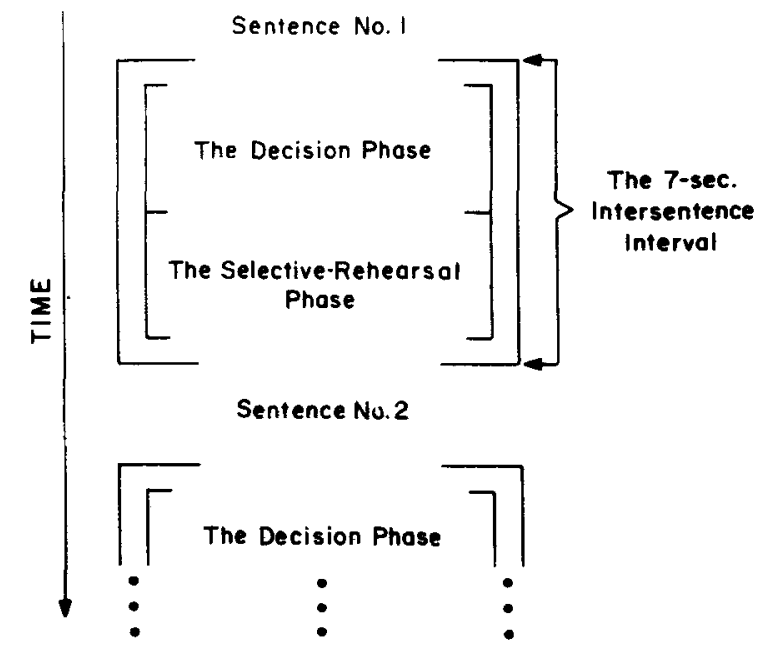

Figure 1. The decision phase and the selective-rehearsal phase of the semantic positive-forgetting task. 
shown that providing subjects with specific learning goals improves the retention of information from prose materials about the goals, but the fate of the material that is irrelevant to the goals seems to be unreliable. Some experiments have shown a decrease in learning for the irrelevant or incidental material, while others have shown an increase in learning about both types of material as a result of the learning goals. The obvious question is whether there are any differences in the learning strategies employed by subjects in goal-directed learning tasks and the study strategy used by subjects in the semantic positive-forgetting task. One procedural difference between the two is that subjects in goaldirected learning experiments are not explicitly told to forget information that is irrelevant to the learning goals, whereas in semantic positive forgetting each sentence is classified as TBR or TBF. Hence, results bearing on the fate of the goal-irrelevant material in goal-directed learning tasks have been unreliable. However, Rothkopf and his associates have found that the irrelevant material is more likely to be remembered if it bears some relation to the goal-relevant material. Geiselman (1974) has observed the same result in the directed forgetting of sentence material. The more the TBF sentences were interconnected with the TBR sentences, the greater was the difficulty in differentially grouping the TBR sentences from the TBF sentences in memory for efficient selective rehearsal.

Geiselman and Riehle (1975) found that presenting the TBF sentences in their logical order, as opposed to in a random order, yielded a greater probability of TBFsentence recall but also a greater probability of TBRsentence recall. The latter result suggests that disclosing to the subject the underlying logical structure of the TBF sentences either (1) leads to faster TBR vs TBF discriminations due to a semantic expectancy or syntax, and therefore allows more time for selective rehearsal of the TBR items (Geiselman, 1975), or (2) simply promotes the formation of a more functionally distinct (noninterfering) grouping of the TBF sentences in memory (Geiselman \& Riehle, 1975). The first hypothesis will be called the expectancy-set hypothesis, after the work of Goodman (1970), and the second hypothesis will be called the set-differentiation hypothesis. Previous research has shown that selective rehearsal of the TBR items is more efficient, with greater ease of differential grouping of the TBR items from the TBF items in memory on the basis of such factors as acoustic discriminability (Block, 1971) or semantic discriminability (Geiselman, 1974). It would be interesting if, contrary to the expectancy-set hypothesis, TBF-sentence connectedness promotes more complete set differentiation of the TBR and TBF items in memory without allowing more of the intersentence interval for TBR selective rehearsal. What governs the efficient functioning of the mechanisms involved in selective forgetting is, of course, an important question for research.

One purpose of the present experiment was to evaluate the expectancy-set hypothesis by measuring the decision times. It could then be determined whether differences in decision times are associated with the TBF-sentence ordering effect on TBR-sentence recall. To obtain decision times of sufficient duration for purposes of the analysis, materials were used that were constructed in metaphorical style (Dooling \& Mullet, 1973). In addition, an attempt was made to extend the analysis to situations where the general theme of the TBF inputs is not known. This was done by providing only half of the subjects with the theme of the TBF sentences. If the subjects are not given the theme of the TBF set of sentences, the set-differentiation hypothesis predicts poorer set differentiation and, therefore, decreased TBR-sentence recall. The expectancy-set, or "extrarehearsal," hypothesis predicts no effect unless the decision times show a concomitant change. It might be expected that the decision times would actually decrease, and thereby increase TBR recall in this condition, since the decisions would probably be made in the context of only one theme, the TBR theme. In either case, it would be interesting to determine if there is a bias toward one type of decision error, that is, calling TBR sentences TBF or calling TBF sentences TBR.

Another purpose of this experiment was to assess possible relationships between decision time and TBRand TBF-sentence recall and recognition. Woodward, Bjork, and Jongeward (1973) found that increasing the time between the presentation of a word and its remember or forget cue facilitated the recognition of the word but had no effect on recall. However, processing during the decision phase of the semantic positive-forgetting task may be somewhat different from the "maintenance" rehearsal during the cue-delay intervals in directedforgetting tasks. Walsh and Jenkins (1973), for example, have shown that orienting tasks which necessitate semantic processing of words result in better recall than nonsemantic tasks regardless of the subject's intention to recall.

A final purpose of the experiment was to compare retrieval and storage differences in the semantic positiveforgetting task by administering a three-alternative forced-choice recognition test for each of the TBR and TBF sentences. Bjork (1972) has suggested that in some cases the positive-forgetting phenomenon may be totally the result of the selective formation of retrieval schemes rather than of differences in storage of the TBR and TBF items. It could not be predicted whether the recognition results would parallel the recall results in the present experiment, but Geiselman (1974) obtained nearly perfect recognition of key words from both TBR- and TBF-cued sentences using the immediate postinput cuing procedure of Woodward and Bjork (1971).

\section{METHOD}

Subjects

The subjects were 128 undergraduate volunteers obtained from introductory psychology courses at Ohio University. 


\section{Materials and Apparatus}

The two sets of sentences that were used as the TBR and TBF items were taken from the Dooling and Mullet (1973) passages entitled "Columbus Discovering America" and "Washing the Dishes." Both of these passages were written in metaphorical style, and eight sentences were chosen from each passage. To determine the most plausible order for each passage, 10 judges were given both sets of eight sentences and were asked to rank each set in its "best" possible order. A coefficient of concordance (Kirk, 1968) was computed from the judges' ranks for each set of sentences as a connectedness index. The coefficient for the "Columbus" passage was $.92(\mathrm{p}<.001)$ and the coefficient for the "Dishes" passage was $.96(p<.001)$.

The two sets of eight sentences were intermixed, with the only restrictions being that no more than two sentences from a given theme could appear in a row and four sentences from each passage must appear in each half of the entire list of 16 sentences. Four different orders of the 16 sentences were generated: one with both sets appearing in their best orders as determined by the judges, one with the "Columbus" passage scrambled, one with the "Dishes" passage scrambled, and one with both passages scrambled. As an example, the ordering for the 16 sentences with both passages in their best order appears below, with the sentences about washing dishes in parentheses.

An egg, not a table, typifies this unexplored planet, he said. (There was a veritable mountain to be conquered.) Hocked gems financed our hero. Scornful laughter had tried to prevent his scheme. (The knife blades were stained after having done their work.) (The warm water foamed around the spot where it fell from above.) Now three sturdy sisters sought proof. (The blue and red striped material moved back and forth rhythmically.) (The egg put up a terrible fight.) They forged along over turbulent peaks and valleys. (The final battle was fought with a pile of blackened steel.) (With the task's completion, the plug was pulled.) Days became weeks as doubters spread fearful rumors about the edge. Bravely he persisted. (Pieces moved gradually and systematically from one side to the other.) At last, welcome winged creatures appeared.

A three-alternative forced-choice recognition test was constructed for each of the 16 sentences by removing a key word from each sentence and generating two plausible alternatives in addition to the correct response. The key words were determined by the judges and the foils were chosen such that they changed the meaning of the sentence. This multiple-choice test was given to 10 judges who had not seen the original sentences to obtain a rate of guessing for the test. The mean score was 5.9 items correct out of 16 , which is not significantly different from chance.

Two other sets of sentences were constructed in metaphorical style to be used on a practice trial. One passage was about a stuntman and the other was about a space shot to the moon. The scrambling of either set or of both sets was done as before to generate the other three presentation orders.

The sentences were presented via a Sony TC-106A tape recorder with 7-sec empty intervals between sentences. The 7-sec intersentence interval size was chosen in accordance with that used in the previous two studies of the semantic positiveforgetting task (Geiselman, 1975; Geiselman \& Riehle, 1975). The decision times were measured via a Marietta 14-1-M reaction time system. Immediately after a sentence was presented, a voice relay, operating directly from the tape recorder when no stimulation occurred, started a timer on the experimenter's display and activated a light on the subject's display, indicating to the subject the start of the decision phase. In addition to the light, the subject's display included two keys, either of which would stop the timer. The two keys were either labeled with the two themes or one was labeled with the TBR theme and one with the words "something else." Each key, when depressed, activated a corresponding light on the experimenter's display.

\section{Design}

The design, collapsed across subjects within cells, was a 2 by 2 by 2 by 2 by 2 by 8 , with the factors being TBR passage ("Columbus" or "Dishes"), TBR-sentence ordering (best order or scrambled), TBF-sentence ordering (best order or scrambled), knowledge of the TBF-sentence theme (known or not known), sentence type (TBR, TBF), and serial position (1-8). The sentence-type and serial-position factors were the only withinsubjects factors.

\section{Procedure}

Each of the 128 subjects was assigned to one of eight groups and was tested individually. After a practice trial using the stuntman and moon shot materials, half of the subjects in each group were told that the "Columbus" passage was TBR and half were told that the "Dishes" passage was TBR. All subjects were given the TBR theme, but each group was presented the TBR sentences either in their best order or in a scrambled order, the TBF sentences either in their best order or in a scrambled order, and was either given the TBF theme prior to the presentation of the sentences or was not given the TBF theme at all. The subjects were told, "This is not a speed test; be sure of each decision before you make it. Even though you must press the appropriate key for each sentence. you will only be tested later for your memory of the sentences about the experimenter-designated TBR theme]. The purpose of the experiment is to see if allowing people to forget some information permits them to remember the other information better."

lmmediately following the presentation of each sentence, the voice relay started the decision-time clock on the experimenter's display and activated the light on the subject's display to alert the subject to being his TBR vs TBF decision. It was acknowledged that some subjects might identify the theme of some sentences correctly before the completion of the sentence, but the purpose of the decision-time recordings was to indicate how much of the intersentence intervals was being used for the purpose of making the TBR vs TBF decision. Hence, such instances would yield rapid decision times. Also, pilot work indicated that some decisions made prematurely, before hearing the entire sentence, were recognized to be incorrect by the subject later on. All subjects made their decisions with their preferred hand, using their second and third fingers.

After all 16 sentences were presented, an unrelated deductive reasoning problem was given to the subjects to complete within $40 \mathrm{sec}$. Then half of the subjects in each group were asked to write down the sentences about the TBR theme and half were asked to write down the sentences about the TBF theme. After 3 min, the subjects were asked to write down the sentences about the remaining theme. Following the recall tests, the subjects were given a recognition sheet containing all 16 sentences, randomized with respect to input serial position, with a key word removed from each sentence. The subject's task was to circle one of three plausible alternatives to fill each blank and a flexible amount of time was allowed for completion of this test.

The subjects who were not given the theme of the TBF sentences received essentially the same instructions, except thcy were told that half the sentences were about some other theme. "something else." Otherwise the procedure was the same. At the end of the experiment, these subjects were asked whether or not they tried to identify the theme of the "something-els" sentences during the presentation of the sentences.

The practice trial for each group was the same as the second trial, including instructions, except that the TBF set of sentences was not tested for recall and there was no recognition test on either the stuntman or moon shot set of sentences. No data were scored from the practice trial. 


\section{Analysis}

There were four dependent variables: log decision time, accuracy of the decision, recall, and recognition. The log transformation was chosen by applying the ratio criterion (Kirk, 1968) to the obtained decision-time data and was conducted to minimize any effects due to some of the within-group distributions of decision times being skewed. Whether a sentence was recalled was determined by two independent judges. One point was awarded by a judge if he thought that the subject had recalled essentially the correct meaning of a sentence and $1 / 2$ point was awarded by a judge if he thought that the subject had partially recalled the sentence. The average of the two judges' scorings of each sentence for each subject was used in the analysis of the recall results. This scoring system was used by Dooling and Mullet (1973), who obtained a significant interjudge difference in retention means but a high interjudge correlation.

If a subject recalled a sentence correctly but under the wrong theme, the sentence was scored as incorrect for purposes of the main recall analysis and the intrusion data were analyzed separately. Each of the four dependent variables was analyzed using a 2 by 2 by 2 by 2 by 2 by 8 analysis of variance, with the factors being the same as outlined in the design section. Then, in a separate analysis, the decision times associated with correct decisions were analyzed independently of the errors, because Pachella (1974) has shown that decision error rates of less than $5 \%$ can significantly alter reaction-time results. This was done by eliminating the serial-position factor from the design and obtaining a mean log decision time for each subject from the correct TBRsentence decisions and for the correct TBF-sentence decisions.

\section{RESULTS}

\section{Sentence Recall}

The correlation between the two judges' scorings of the sentences recalled by each subject was $.72(\mathrm{p}<.001)$. The analysis of variance conducted on the average score for each subject for each sentence showed significant main effects of sentence type (TBR > TBF) and knowledge of the TBF theme, but the Sentence Type by Knowledge of the TBF Theme interaction effect was also significant $[\mathrm{F}(1,112)=16.43, \mathrm{MSe}=.09, \mathrm{p}<.001]$. A Cicchetti test (Cicchetti, 1972) conducted on the interaction effect showed that knowing the TBF theme increased TBF-sentence recall $(p<.01)$ but did not affect TBR-sentence recall. Hence, understanding the intended meanings of the TBF sentences did not influence the accessibility of the TBR sentences. This result would appear to be inconsistent with the setdifferentiation hypothesis.

The main effect of TBF-sentence ordering was significant $[\mathrm{F}(1,112)=4.06, \mathrm{MSe}=.13, \mathrm{p}<.05]$, with more sentences recalled when the TBF sentences were presented in their logical order. As was found by Geiselman and Riehle (1975), the TBF Ordering by Sentence Type interaction effect was not significant $(F<1)$. Presenting the TBF sentences in their best order increased both TBR- and TBF-sentence recall. The TBR-sentence ordering effect was also significant $[F(1,112)=15.87$, $\mathrm{MSe}=.13, \mathrm{p}<.001]$, with more sentences recalled when the TBR sentences were presented in their best order. A somewhat surprising finding was that the TBR Ordering by Sentence Type interaction effect was not
Table 1

Mean Number of Sentences Recalled as a Function of Presentation Condition, Sentence Type, and Knowledge of the TBF Theme

\begin{tabular}{|c|c|c|c|c|c|c|c|}
\hline \multirow{2}{*}{$\begin{array}{l}\text { Sentence Type- } \\
\text { Knowledge of } \\
\text { TBF Theme† }\end{array}$} & \multicolumn{7}{|c|}{ Presentation Condition* } \\
\hline & $\begin{array}{c}\text { TBR } \\
\mathrm{S}\end{array}$ & $\underset{\mathrm{S}}{\mathrm{TBF}}$ & $\begin{array}{c}\text { TBR } \\
\mathrm{O}\end{array}$ & $\begin{array}{c}\text { TBF } \\
\text { S }\end{array}$ & $\begin{array}{c}\text { TBR } \\
\mathrm{S}\end{array}$ & $\begin{array}{c}\text { TBF } \\
\mathrm{O}\end{array}$ & $\begin{array}{cc}\text { TBR } & \text { TBF } \\
\mathbf{O} & 0\end{array}$ \\
\hline TBF & 2. & .2 & & .7 & 2 & .4 & 3.3 \\
\hline TBF $-K$ & & .9 & & .7 & & .6 & 2.1 \\
\hline TBR-NK & & .4 & & 8 & & .3 & 3.0 \\
\hline TBF-NK & & .5 & & .6 & & .4 & .4 \\
\hline
\end{tabular}

" $S=$ scrambled,$O=$ ordered

$\dagger_{K}=T B F$ theme known, NK $=T B F$ theme not known

significant $(F<1)$. Presenting the TBR sentences in their logical order not only increased TBR-sentence recall but also increased TBF-sentence recall. Using less ambiguous sentences as the stimulus materials, Geiselman and Riehle (1975) found that presenting the TBR sentences in their best order decreased TBF-sentence recall.

In addition, the TBR Ordering by Knowledge of the TBF Theme by Sentence Type interaction was significant $[F(1,112)=4.02, \mathrm{MSe}=.09, \mathrm{p}<.05]$, as was the TBF Ordering by Knowledge of the TBF Theme interaction effect $[\mathrm{F}(1,112)=4.65, \mathrm{MSe}=.13, \mathrm{p}<.05]$. Cicchetti tests on these interactions showed that when the TBF-sentence theme was not known, presenting the TBR sentences in their best order did not increase TBFsentence recall and presenting the TBF sentences in their best order did not increase either TBR-or TBF-sentence recall. These results indicate that knowledge of the TBF theme is an important variable in observing the ordering effects. The mean number of TBR and TBF sentences recalled under the four presentation conditions when the TBF-sentence theme was known and was not known are shown in Table 1.

The analysis of variance conducted on the mean number of TBR sentences which were recalled as TBF and the mean number of TBF sentences which were recalled as TBR for each subject showed that there were more intrusions of both types when the TBF theme was not known $[\mathrm{F}(1,112)=4.81, \mathrm{MSe}=.14, \mathrm{p}<.05]$. There was an average of .21 intrusions per subject when the TBF-sentence theme was known as compared to .31 when the TBF-sentence theme was not known. Hence, neither intrusion rate was influenced by either TBR- or TBF-sentence ordering.

\section{Key-Word Recognition}

The analysis of variance conducted on the recognition data showed a significant main effect of sentence type (TBR > TBF) but the Sentence Type by Knowledge of the TBF Theme interaction effect was also significant $[F(1,112)=6.25, \mathrm{MSe}=.12, \mathrm{p}<.05]$. A Cicchetti test conducted on the interaction indicated that TBRsentence recognition was greater than TBF-sentence 
Table 2

Mean Number of Key-Word Recognitions as a Function of Presentation Condition, Sentence Type, and Knowledge of the TBF Theme

\begin{tabular}{|c|c|c|c|c|c|c|c|}
\hline \multirow{2}{*}{$\begin{array}{l}\text { Sentence Type- } \\
\text { Knowledge of } \\
\text { TBF Theme- }\end{array}$} & \multicolumn{7}{|c|}{ Presentation Condition* } \\
\hline & $\begin{array}{c}\text { TBR } \\
S\end{array}$ & $\begin{array}{c}\text { TBF } \\
\mathrm{S}\end{array}$ & $\begin{array}{c}\text { TBR } \\
\text { O }\end{array}$ & $\begin{array}{c}\text { TBF } \\
\mathrm{S}\end{array}$ & $\begin{array}{c}\text { TBR } \\
\mathrm{S}\end{array}$ & $\begin{array}{c}\text { TBF } \\
\mathrm{O}\end{array}$ & $\begin{array}{cc}\text { TBR } & \text { TBF } \\
\mathrm{O} & \mathrm{O}\end{array}$ \\
\hline TBR & & & 7. & 9 & 7. & 0 & 7.4 \\
\hline TE & & & 7. & & & & 7.4 \\
\hline TBR $-\mathrm{NK}$ & 7. & & 7. & & 7. & & 7.1 \\
\hline $\mathrm{TBF}-\mathrm{NK}$ & 6. & 2 & 6. & & 6. & & 6.0 \\
\hline
\end{tabular}

$* S=$ scrambled, $O=$ ordered

${ }^{\prime} K=T B F$ theme know' $. N K=T B F$ theme not known

recognition only when the TBF-sentence theme was not known $(p<.05)$. Hence, it appears that there was a decrease in storage or processing of the TBF sentences as well as in accessibility when the TBF-sentence theme was not given to the subjects. Also, these data indicate that the difference between TBR- and TBF-sentence recall when the TBF-sentence theme was known was not due to a difference in storage of the two sentence types.

The main effect of TBR-sentence ordering was significant, but the TBR Ordering by Knowledge of the TBF Theme interaction effect was also significant $[F(1.112)=3.97, \mathrm{MSe}=.16, \mathrm{p}<.05]$. A Cicchet ti test indicated that TBR-sentence ordering increased recognition only when the TBF-sentence theme was known $(p<.05)$. Hence, if the TBF-sentence theme was known. presenting the TBR sentences in their best order tended to facilitate the storage as well as the retrieval of both sentence types. The mean number of correct recognitions of key words from the TBR and TBF sentences under the four presentation conditions when the TBF theme was known and was not known are shown in Table 2.

\section{Decision Time}

An analysis of variance was first conducted on the $\log$ decision times regardless of the accuracy of each decision. The analysis of variance showed that the TBR Ordering by Knowledge of the TBF Theme interaction effect was significant $[F(1.112)=4.21$, MSe $=1.36$, $\mathrm{p}<.05]$. A Cicchetti test indicated that presenting the TBR sentences in their logical order decreased decision times if the TBF-sentence theme was not known $(\mathrm{p}<.05)$, but actually increased decision times if the TBF-sentence theme was known $(p<.05)$. Hence, the subjects who were given the TBF theme must have used a different sentence-interpretation strategy than the subjects who were not given the TBF theme. The TBRsentence ordering factor did not interact with the sentence-type factor.

The TBF Ordering by Knowledge of the TBF Theme interaction effect was also significant $[F(1,112)=4.17$. $\mathrm{MSe}=1.36, \mathrm{p}<.05]$. A Cicchetti test indicated that decision times were shorter when the TBF sentences were presented in their logical order, but only if the TBF-sentence theme was known $(\mathrm{p}<.05)$. The means corresponding to the two TBF-sentence ordering conditions were identical. The latter result suggests that the subjects who were not given the TBF theme were not attempting to liypothesize a theme for the TBF sentences or even to detect the TBF sentences on the basis of the serial-position cues in those sentences. In fact, only 9 of these 64 subjects reported that they had actively tried to guess the TBF theme, and the 9 subjects were evenly distributed among the four presentation conditions.

As was the case with TBR-sentence ordering, the TBF-sentence ordering factor did not interact with the sentence-type factor. Hence, when the TBF-senterce theme was known, either TBR- or TBF-sentence ordering affected both TBR- and TBF-sentence decision times. These results suggest that when the TBF theme was known, the subjects made the TBR vs TBF decisions in the contexts of both themes. The mean decision times in seconds, obtained by taking the antilogs of the mean log decision times, for the TBR and TBF sentences as a function of presentation condition and knowledge of the TBF theme are shown in Table 3.

An analysis of variance was also conducted on those decision times that were associated with correct decisions. A mean log decision time was computed from the correct decisions for each subject for the TBR sentences and for the TBF sentences. This analysis showed the same effects to be significant as in the previous analysis. However, a $t$ test conducted on the grand means corresponding to correct and incorrect decisions showed that decision times associated with correct decisions were faster than decision times associated with incorrect decisions $[\mathrm{t}(126)=11.70, \mathrm{p}<.001]$. The mean decision time for the correct decisions was $1.38 \mathrm{sec}$. as compared to $1.75 \mathrm{sec}$ for the incorrect decisions.

Decision time and recall. To determine if there were any systematic relationships between log decision time for correct decisions and sentence recall, a correlation between these two variables was computed for each subject for each sentence type. After a Fisher's z transformation, these correlations were analyzed using a 2 by 2 by 2 by 2 by 2 analysis of variance, with the

Table 3

Mean Decision Time (in Seconds) as a Function of Presentation Condition, Sentence Type, and Knowledge of the TBF Theme

\begin{tabular}{|c|c|c|c|c|c|c|c|}
\hline \multirow{2}{*}{$\begin{array}{l}\text { Sentence Type- } \\
\text { Knowledge of } \\
\text { TBF Theme- }\end{array}$} & \multicolumn{7}{|c|}{ Presentation Condition* } \\
\hline & $\begin{array}{c}\text { TBR } \\
\mathbf{S} \\
\end{array}$ & $\begin{array}{c}\text { IBF } \\
\mathrm{S}\end{array}$ & $\begin{array}{c}\text { TBR } \\
\mathrm{O}\end{array}$ & $\begin{array}{c}\text { TBF } \\
\mathrm{S}\end{array}$ & $\begin{array}{c}\text { TBR } \\
\text { S }\end{array}$ & $\begin{array}{c}\text { TBF } \\
\mathbf{O}\end{array}$ & $\begin{array}{cc}\text { TBR } & \text { TBF } \\
\mathrm{O} & \mathrm{O} \\
\end{array}$ \\
\hline TBR $-\mathbf{K}$ & & 33 & & 81 & & .23 & 1.47 \\
\hline $\mathrm{TBF}-\mathrm{K}$ & & 34 & 1. & 85 & & .15 & 1.43 \\
\hline TBR-NK & & 72 & & 28 & & .55 & 1.37 \\
\hline $\mathrm{TBF}-\mathrm{NK}$ & & 68 & 1. & 27 & & .56 & 1.41 \\
\hline
\end{tabular}

* $S=$ scrambled. $O=$ ordered

$\div K=T B F$ the'me known. IK = TBF the'nc not known 


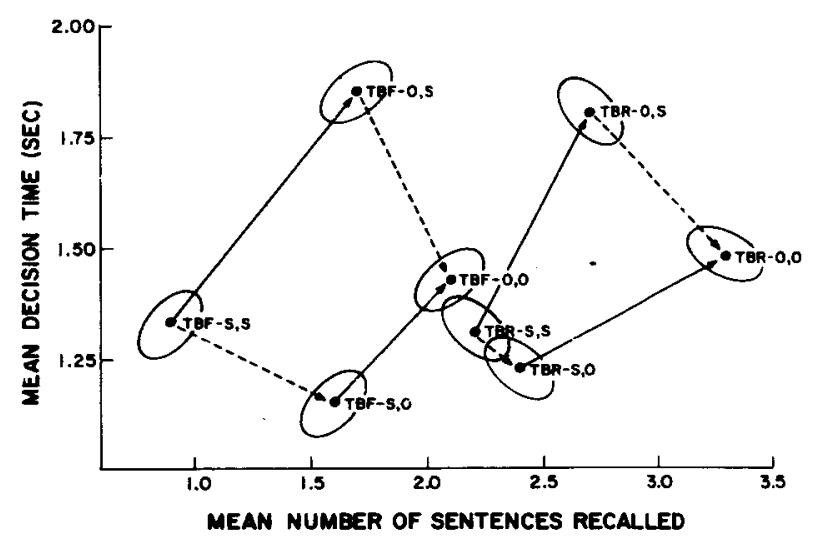

Figure 2. The relationship between decision time and sentence recall when the TBF theme was known as a function of sentence type and TBR-and TBF-sentence ordering. The ellipses are used to illustrate the slopes of the respective regression lines.

factors being the same as outlined in the Design section. The serial-position factor was necessarily deleted. This analysis showed that the only significant effect was the main effect of sentence type $[F(1,112)=15.47$, $\mathrm{p}<.001]$. The mean correlation was -.32 for the TBR sentences and +.35 for the TBF sentences. Both of these correlations are significantly different from zero $(p<.001)$. Hence, shorter decision times are associated with greater TBR-sentence recall, whereas longer decision times are associated with greater TBF-sentence recall.

The relationship between sentence recall and decision time when the theme of the TBF sentences was known is shown in Figure 2 as a function of sentence type and sentence ordering. Each point in Figure 2 is labeled TBR or TBF, and the first ordering symbol following the label refers to the ordering of the TBR sentences $(\mathrm{O}=$ ordered, $\mathrm{S}=$ scrambled $)$. The second ordering symbol refers to the ordering of the TBF sentences. The solid lines represent the effects of presenting the TBF sentences in their best order while holding the order of the TBF sentences constant, and the broken lines represent the effects of presenting the TBF sentences in their best order while holding the order of the TBR sentences constant. The small ellipses around each point are used to illustrate the average slopes of the respective regression lines and are not actual representations of the obtained distributions of values. Figure 2 demonstrates that the within-cell relationships between decision time and sentence recall are consistent with the TBR-ordering effect on TBF-sentence recall (see the solid lines on the left side of the figure) and with the TBF-ordering effect on TBR-sentence recall (see the broken lines on the right side of the figure). The within-cell relationships between decision time and recall appear to be unrelated to the TBF-ordering effect on TBF-sentence recall and the TBR-ordering effect on TBR-sentence recall; that is, the regression lines are nearly perpendicular to the broken lines on the left side of the figure and to the solid lines on the right side of the figure. Hence, only the facilita- tive effects of presenting one passage in its best order on the recall of the other passage are consistent with the general relationships between decision time and recall.

Decision time and recognition. The correlations between log decision time for correct decisions and keyword recognition were analyzed in the same manner as above. This analysis showed no significant effects, but the grand mean was significantly greater than zero, +.31 $[F(1,112)=43.11, p<.001]$. Hence, longer decision times were associated with both greater TBR-and TBFsentence key-word recognition. As noted earlier, presenting the TBR sentences in their best order facilitated recognition performance for both the TBR and TBF sentences when the TBF theme was known. However, TBR-sentence ordering also increased decision times. Perhaps decision time served as a mediating variable in the TBR-sentence ordering effect on recognition.

\section{Decision Accuracy}

The analysis of variance conducted on the decision accuracy data showed significant main effects of sentence type and knowledge of the TBF theme, but the Sentence Type by Knowledge of the TBF Theme interaction effect was also significant $[F(1,112)=17.20$, $\mathrm{MSe}=.13, \mathrm{p}<.001]$. A Cicchetti test indicated that decision accuracy for the TBR sentences was smaller than decision accuracy for the TBF sentences, but only if the TBF theme was not known $(p<.01)$. This was due to a decrease in decision accuracy for the TBR sentences. Since decision accuracy for the TBF sentences did not show a concomitant increase, the subjects must have set their TBR vs TBF response criterion in relation to the distribution of TBF sentences, on a scale of "to be rememberedness." When the TBF theme was not known, the subjects judged the TBR sentences as having less association with the TBR theme, relative to the TBF sentences, than if the TBF theme was known. This suggests that some form of exhaustive interpretation of each sentence occurred when the TBF theme was known.

Neither TBR-sentence ordering nor TBF-sentence ordering affected decision accuracy. Hence, the effects of sentence ordering on sentence recall were not due to changes in theme-membership discriminability or changes in decision bias. The mean number of correct

Table 4

Mean Number of Correct Decisions as a Function of Presentation Condition, Sentence Type, and Knowledge of the TBF Theme

\begin{tabular}{|c|c|c|c|c|c|c|c|}
\hline \multirow{2}{*}{$\begin{array}{l}\text { Sentence Type- } \\
\text { Knowledge of } \\
\text { TBF Theme }\end{array}$} & \multicolumn{7}{|c|}{ Presentation Condition* } \\
\hline & $\begin{array}{c}\text { TBR } \\
\text { S }\end{array}$ & $\begin{array}{c}\text { TBF } \\
\text { S }\end{array}$ & $\begin{array}{c}\text { TBR } \\
\mathrm{O}\end{array}$ & $\begin{array}{c}\text { TBF } \\
\text { S }\end{array}$ & $\begin{array}{c}\text { TBR } \\
\text { S }\end{array}$ & $\begin{array}{c}\text { TBF } \\
\mathrm{O}\end{array}$ & $\begin{array}{cc}\text { TBR } & \text { TBF } \\
\mathrm{O} & \mathrm{O} \\
\end{array}$ \\
\hline TB & 6 & .9 & 7. & .0 & 6 & 8 & 7.2 \\
\hline TBF & 6. & 9 & 7. & .0 & & & 7.0 \\
\hline TBR-NK & 5 & 6 & & .6 & & & 6.4 \\
\hline $\mathrm{TBF}-\mathrm{NK}$ & 6. & .9 & & .0 & & & 6.6 \\
\hline
\end{tabular}

${ }^{*} S=$ scrambled,$O=$ ordered

$+K=T B F$ theme known, $N K=T B F$ theme not known 
decisions for the TBR and TBF sentences as a function of presentation condition and knowledge of the TBF theme is shown in Table 4.

\section{DISCUSSION}

With respect to the original purposes for conducting this experiment, the data were quite revealing. First, the expectancy-set hypothesis for the TBF-sentence ordering effect on TBR-sentence recall was supported. Second, the decision-time data indicated that there was an interaction between processing difficulty and processing intent with respect to sentence recall; therefore, processing during the decision phase seems to be different from processing during the cue-delay interval in directedforgetting tasks. Also, differences in decision time, but not in sentence ordering, were associated with key-word recognition. Third, analyses of all four dependent variables suggested that knowledge of the TBF theme is an important factor in the semantic positive-forgetting task. Each of these problems is discussed below.

\section{TBF Theme Known}

Sentence ordering and recall. As reported by Geiselman (1975) and Geiselman and Riehle (1975), presenting the TBF sentences in their logical order increased recall for the TBR sentences as well as for the TBF sentences. However, the general relationship between decision time and TBR-sentence recall $(\mathrm{r}=-.32)$ was consistent with the TBF-sentence ordering effect on TBR-sentence recall (see Figure 2). Therefore, this recall effect may not be due to a more functionally distinct logical structure for the TBF sentences in long-term store but rather to more functional selective-rehearsal time for the TBR sentences. Also, since knowledge of the TBF theme did not affect the overall probability of TBR-sentence recall, the set differentiation hypothesis is probably not correct. Surely, knowing the theme of the TBF passage should improve the set differentiation in memory if selective rehearsal were not the prepotent factor. Knowing the TBF theme did not yield an overall decrease in decision times to allow for more TBRsentence selective rehearsal.

Further, the facilitative effect of TBR-sentence logical ordering on TBF-sentence recall was consistent with the general relationship between decision time and TBF. sentence recall $(\mathrm{r}=+.35$, see Figure 2$)$. Presenting the TBR sentences in their best order yielded longer decision times which may have eliminated the need for additional processing of the TBF sentences for their retrieval later. This conclusion seems plausible since Geiselman and Riehle (1975) found that, when the meaning of each sentence was readily apparent in the semantic positiveforgetting task, presenting the TBR sentences in their best order decreased TBF-sentence recall. This was interpreted as being the result of fewer draws of the TBF sentences from memory during TBR-sentence selective rehearsal if the subjects had a logical structure for the TBR set of sentences to use as a rehearsal scheme.

TBR-sentence ordering facilitated TBR-sentence recall and TBF-sentence ordering facilitated TBFsentence recall, but these effects were not consistent with the generai relationships between decision time and recall (see Figure 2). This result supports Kintsch's (1974) concept of macrostructure of a passage. The same sets of sentences presented in random orders may have appeared to merely be catalogs of expressions about Columbus and washing dishes. Therefore, a randomly presented set of sentences was probably stored in memory in a less organized fashion than a logically ordered set of sentences, since the "story-like" nature of the passage may not have been recognized. By replacing definite articles with indefinite articles, de Villiers (1974) found that subjects who recalled a passage as a story recalled more sentences than subjects who perceived the passage as a series of unrelated sentences.

Decision time and retention. Shorter decision items were associated with a greater probability of TBRsentence recall, but longer decision times were associated with a greater probability of TBF-sentence recall. Apparently, ease of comprehension facilitates recall if there is intent to remember the sentence, but extended semantic processing facilitates recall if there is no intent to remember the sentence. The latter result is consistent with the levels-of-processing notion that, if a stimulus is "difficult to process, more analyses are carried out and a richer memory trace results" (Lockhart, Craik, \& Jacoby, 1976, p. 79). Processing during the decision phase of the semantic positive-forgetting task appears to be somewhat different from the "maintenance rehearsal" during the cue-delay interval in directed-forgetting tasks that has no effect on recall (Woodward et al., 1973). Sequential organization for the TBF sentences also improved recall of the TBF sentences, but with shorter decision times. This result is consistent with Lockhart et al.'s second kind of processing "depth" in that "structural descriptions at any level are as much a product of expectancies and past learning as they are products of the current stimulus input ... since they have been largely performed in anticipation" (p. 78).

The finding that longer decision times were associated with a higher level of recognition performance for both sentence types is consistent with the results of the experiment conducted by Woodward et al. (1973). They found that increasing the time between the presentation of a word and its remember or forget cue facilitated the recognition of the word. Perhaps with longer decision times, there is a greater probability that the specific words in the sentence are stored in memory. The only significant effect of sentence ordering on recognition was the facilitative effect of TBR-sentence ordering on both TBR and TBF recognition. However, TBR-sentence ordering also increased the decision times. Therefore, probably none of the sentence-ordering effects on recall can be attributed to differences in storage of the sentences. 
Making the decisions. If the subject knows the general theme of the TBF sentences, it appears that the subject interprets each sentence in the context of both themes. The logical ordering of the sentences referring to the TBF theme affected the decision times for the sentences referring to the TBR theme and vice versa. Further, presenting the TBR sentences in their best order decreased decision times when the TBF theme was not known but increased decision times when the TBF theme was known. This is compelling evidence that some form of exhaustive interpretation occurred when the TBF theme was known. The reason why presenting the TBR sentences in their logical order increased decision times when the TBF theme was known is not apparent and requires further research.

\section{TBF Theme Not Known}

Knowledge of the TBF theme did not affect TBRsentence recall or key-word recognition, but both TBFsentence recall and key-word recognition performance decreased when the TBF theme was not known. The latter findings support Dooling and Mullet's (1973) conclusion that the locus of the thematic effect in prose retention is at the point of storage of the material rather than merely at retrieval. The only sentenceordering effect that was significant was the TBRsentence ordering effect on TBR-sentence recall. This indicates that knowledge of the TBF theme is important in observing the ordering effects. Presenting the TBF sentences in their logical order did not decrease decision times to allow more selective rehearsal of the TBR sentences and did not disclose the underlying structure of the TBF sentences for greater TBF recall. Presenting the TBR sentences in their logical order did not lead to longer initial processing of the TBF sentences, which was apparently a factor in producing the TBR-ordering effect on TBR recall when the TBF theme was known.

It also appears that the TBR vs TBF decision for each sentence is made solely on the basis of an attempted interpretation in the context of the TBR theme. The subjects reported that they did not attempt to guess the theme of the TBF sentences, and presenting the TBF sentences in their best order did not affect TBR- or TBF-sentence decision times. Further, there was a bias toward calling the TBR sentences TBF. If a TBR sentence could not be incorporated into the TBR theme, the subjects decided that the sentence was TBF, a member of the "something-else" theme. This singleinterpretation process was no faster, overall, than the exhaustive-interpretation process that was apparently employed when both themes were known. Perhaps the subjects were not willing to make rapid decisions in this condition since each sentence could have been a member of any number of themes.

The general importance of knowing the TBF theme in semantic positive forgetting can be seen in the context of the "cocktail-party problem" analogy (Geiselman, 1975). Hearing a series of irrelevant inputs in a logical sequence, as opposed to in an illogical sequence, does not appear to facilitate the encoding of the mainstream of a conversation unless the general theme of the irrelevant ideas is known. In fact, if the theme of the irrelevant ideas is not known, some ambiguous inputs that are intended to be part of the mainstream are likely to be ignored as irrelevant statements.

\section{REFERENCES}

Bjork, R. A. Positive forgetting, the non-interference of items intentionally forgotten. Journal of Verbal Learning and Verbal Behavior, 1970, 9, 255-268.

BJORK, R. A. Theoretical implications of directed forgetting. In A. W. Melton \& E. Martin (Eds.), Coding processes in human memory. Washington, D.C: Winston, 1972.

BLock, R. A. Effects on instructions to forget in short-term memory. Journal of Experimental Psychology, 1971, 89, 1-9.

Cicchetri, D. V. Extension of multiple-range tests to interaction tables in the analysis of variance: A rapid approximate solution. Psychological Bulletin, 1972, 77, 405-408.

DE VILLIERS, P. A. Imagery and theme in recall of connected discourse. Joumal of Experimental Psychology, 1974, 103, 263-268.

Dooling, D. J., \& Mullet, R. L. Locus of thematic effects in retention of prose. Journal of Experimental Psychology, 1973. 97, 404-406.

Geiselman, R. E. Positive forgetting of sentence material. Memory \& Cognition, 1974, 2, 677-682.

Geiselman, R. E. Semantic positive forgetting: Another cocktail party problem. Journal of Verbal Learning and Verbal Behavior, $1975,14,73-81$.

Geiselman, R. E., \& Riehle, J. P. The fate of to-be-forgotten sentences in semantic positive forgetting. Bulletin of the Psychonomic Society, 1975, 6, 19-21.

Goodman, K. S. Reading: A psycholinguistic guessing game. In K. Gunderson (Ed.), Language \& reading. Washington, D.C: Center for Applied Linguistics, 1970.

JAMEs, W. The principles of psychology (Vol. 2). New York: H. Holt, 1890.

KINTSCH, W. The representation of meaning in memory. Hillsdale, N.J: Lawrence Erlbaum, 1974.

KIRK, R. E. Experimental design: Procedures for the behavioral sciences. Belmont, Calif: Wadsworth, 1968.

Lockhart, R. S., CRaIK, F. I. M., \& Jacoby, L. Depth of processing, recognition, and recall. In J. Brown (Ed.), Recall and recognition. New York: John Wiley, 1976.

Pachella, R. G. The interpretation of reaction time in information processing research. In B. H. Kantowitz (Ed.), Human information processing: Tutorials in performance and cognition. Hillsdale, N.J: Lawrence Erlbaum, 1974.

Riвot, T. A. Diseases of memory: An essay in the positive psych. ology. New York: Appleton-Centure-Crofts, 1882.

RoThKOPF, E. Z. Writing to teach and reading to learn: A perspective on the psychology of written instruction. Seventy-fifth yearbook of the national society for the study of education. Chicago: University of Chicago Press, 1976. Pp. 9i-129.

WALSh, D. A., \& JENKINS, J. J. Effects of orienting tasks on free recall in incidental learning: "Difficulty," "effort," and "process" explanations. Journal of Verbal Learning and Verbal Behavior, 1973, 12, 481-488.

Woodward, A. E., \& Bjork, R. A. Forgetting and remembering in free recall: Intentional and unintentional. Journal of Experimental Psychology, 1971, 89, 109-116.

Woodward, A. E., Bjork, R. A., \& Jongew ARd, R. H., JR. Recall and recognition as a function of primary rehearsal. Journal of Verbal Learning and Verbal Behavior, 1973, 12, 608-617.

(Received for publication December 9, 1976; accepted February 15, 1977.) 